\title{
THE NSW ABORIGINAL VASCULAR HEALTH PROGRAM
}

Margaret Scott

Public Health Division

NSW Department of Health

The NSW Department of Health's Aboriginal Vascular Health Program is responsible for implementing those components of the NSW Aboriginal Health Strategic Plan that relate to cardiovascular disease, renal disease, diabetes, and stroke. ${ }^{1}$ This article describes the Program and the strategies being implemented to prevent and manage vascular disease in indigenous people in NSW.

\section{RATIONALE FOR A VASCULAR HEALTH PROGRAM}

Both diabetes and cardiovascular disease are national health priorities. It is well recognised that indigenous people suffer an excessive burden of vascular disease. ${ }^{2,3,4,5,6,7}$ Vascular disease is the primary cause of preventable mortality and morbidity in indigenous Australians, who have a far higher prevalence of cardiovascular disease, renal disease, diabetes, and stroke, compared with non-indigenous populations. ${ }^{2-8}$

Further, indigenous Australians have higher rates of preventable risk factors such as:

- central obesity;

- poor diet and nutrition;

- low levels of physical activity;

- smoking;

- high-risk alcohol use. ${ }^{3}$

There is increasing evidence that social determinants are as important as physiological risk factors in the aetiology of vascular disease. ${ }^{9,10,11}$ Historical precursors, cultural circumstances, and the socioeconomic and environmental contexts in which indigenous people live, create risk conditions and predispose to behaviours that lead to increased risk of vascular diseases (Figure 1). These factors also militate against people managing established disease in ways that improve health outcomes. In addition, excess mortality in people with diabetes increases with social disadvantage. ${ }^{12}$ Recent work in the Northern Territorywhich is reported elsewhere in this issue of the Bulletin-is describing the causal pathway between socioeconomic disadvantage and end stage renal failure. ${ }^{13}$

\section{Common behavioural and physiological risk factors for vascular disease}

Diabetes and heart disease share common aetiological risk behaviours and physiological risk factors. The 'metabolic syndrome', whereby a cluster of metabolic risk factors increases the likelihood of developing cardiovascular disease, is particularly significant in indigenous populations. ${ }^{14,15,16,17}$ Cardiovascular risk is multiplied by the coexistence of several factors. Also diabetes is an independent risk factor for heart disease and the risk of developing heart disease is $2-3$ times higher for people with diabetes. ${ }^{2}$

\section{Advantages of a vascular health approach}

Primary prevention messages relating to health and lifestyle are the same for diabetes and heart disease. Common lifestyle adjustments and some common medical treatments are necessary to manage and prevent progression of these vascular diseases. Programs that integrate clinical risk reduction and primary prevention strategies have the potential to delay the onset of vascular disease in indigenous populations and to minimise adverse health outcomes for those with established disease. An integrated health and lifestyle approach is consistent with the strategic direction of national and state policies for other populations, and is in keeping with holistic indigenous conceptions of health and illness. ${ }^{18,19}$

\section{The evidence base for management of vascular} disease

There is well-established evidence for the efficacy of clinical interventions in improving health outcomes in vascular disease, ${ }^{20,21}$ but there is limited understanding of how these can be successfully implemented within indigenous populations.

Studies from communities in the Tiwi and Torres Strait Islands have demonstrated vascular health gains through multifaceted community-based interventions focused on primary health care services. ${ }^{22,23}$ Common elements of these programs have included:

- education and support for local indigenous health workers working in multi-disciplinary care teams;

- implementation of registration and recall systems and standardised clinical protocols;

- customised support for people to manage their disease.

These studies confirm similar findings with comparable populations overseas. ${ }^{24}$

\section{ABORIGINAL VASCULAR HEALTH PROGRAM}

The aim of the Aboriginal Vascular Health Program is to work in collaboration with relevant organisations and service providers to improve the provision of high-quality prevention and care services and programs, which promote the vascular health of Aboriginal and Torres Strait Islander people in New South Wales.

The strategic foci of the Program include :

- research and development;

- workforce development;

- service development;

- resource development;

- monitoring and evaluation;

- coalition building;

- communication and information dissemination. 


\section{FIGURE 1}

\section{FACTORS CONTRIBUTING TO DIABETES AND CARDIOVASCULAR DISEASE IN ABORIGINAL AND TORRES}

STRAIT ISLANDER PEOPLE

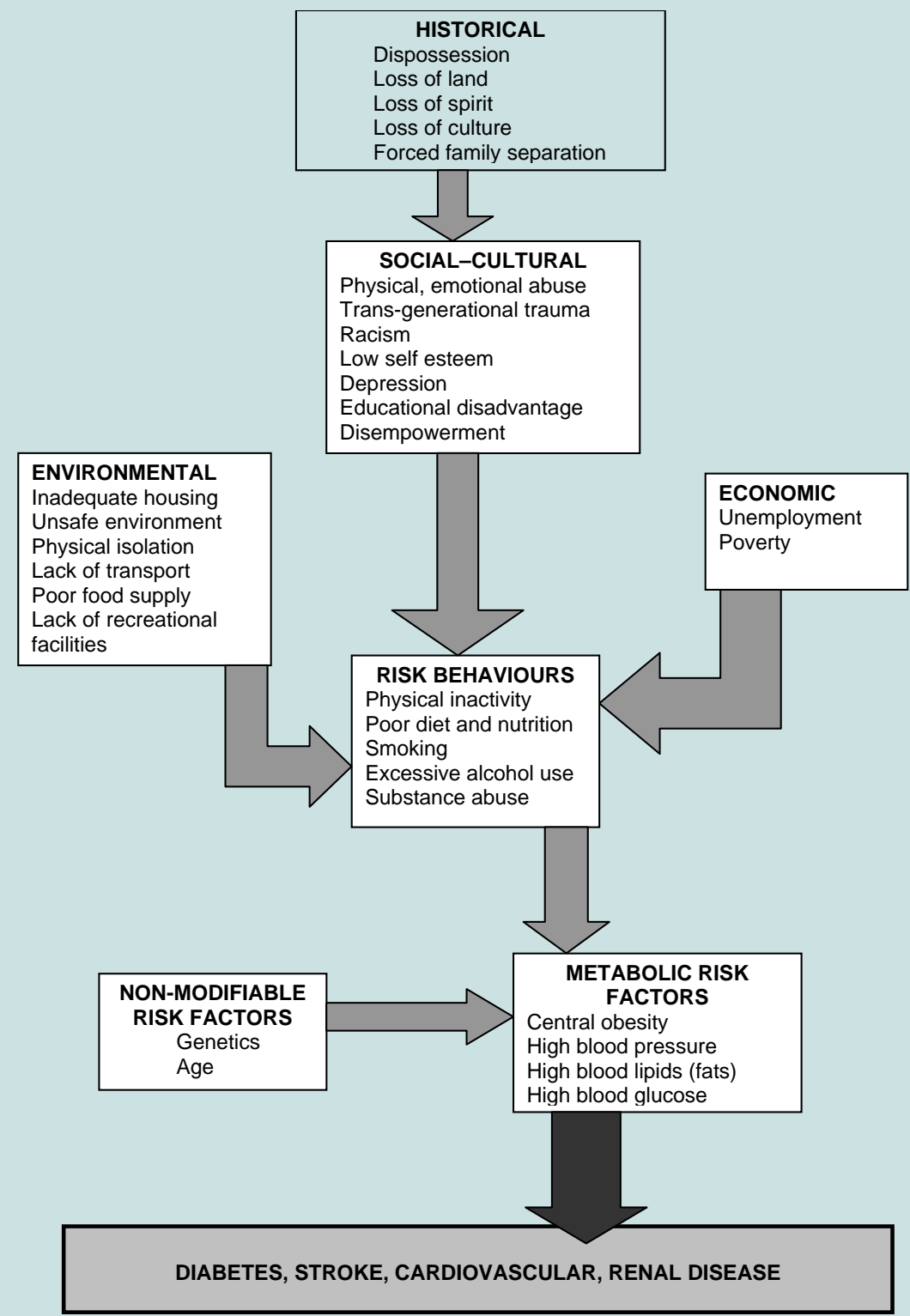

Current activities of the Program include:

\section{Demonstration site projects}

In consultation with local Aboriginal Health Partnerships, 14 demonstration site projects are implementing models of service delivery tailored to local resources and identified needs and priorities. Projects are based in the area health services across NSW and run over 1-2 years. Ongoing funding will be available for projects with demonstrated effectiveness.

\section{Aboriginal Vascular Health Network}

An Aboriginal Vascular Health Network has been established to promote information exchange and resource dissemination and to foster professional development opportunities for workers involved in vascular health. A quarterly bulletin Vascular Health Matters is produced and distributed to more than 200 network members.

\section{Aboriginal Vascular Health Resource Catalogue}

The Aboriginal Vascular Health Resource Catalogue is an annotated list of available vascular health resources for Australian health professionals, ${ }^{25}$ including training and patient education manuals, Web sites, and useful contact information.

\section{Chronic and Complex Care Program}

The Program is working with cardiovascular projects within the area health services sponsored by the NSW Chronic and Complex Care Program, to enhance their 
appropriateness and accessibility for indigenous communities.

\section{Other activities}

The Program is undertaking collaborative ventures with area health services, Aboriginal health services, and nongovernment organisations, to develop resources and clinical guidelines, provide training opportunities, and to provide advice and technical support for regional initiatives.

\section{CONCLUSION}

Important opportunities for the Program lie in building the capacity of local service providers and ensuring optimal utilisation of local resources through harnessing the expertise of health professionals, organisations, and community members. A major challenge lies in building the evidence base of what works in improving vascular health outcomes for indigenous people. A longer-term outlook and commitment of resources is essential as changes in health status are likely to be slow. However, in the short and medium term, improvements in service delivery and workforce capacity through Program activities are expected; monitoring and evaluation will assess the outcomes and effectiveness. An Aboriginal Vascular Health Indicators Framework is being developed to track progress in improving Aboriginal vascular health across NSW. This will incorporate a new set of indicators of local capacity and intervention to monitor changes that are likely to affect longer term improved health outcomes. A future issue of the Bulletin will report on this framework.

\section{REFERENCES}

1. NSW Department of Health. NSW Aboriginal Health Strategic Plan. Sydney: NSW Department of Health, 1999. www.health.nsw.gov.au/health-public-affairs/ahealth/ astratplan.htm.

2. Australian Institute of Health and Welfare, National Heart Foundation, National Stroke Foundation. Heart, Stroke and Vascular Disease. Australian Facts 2001. Canberra: Australian Institute of Health and Welfare, 2001.

3. Australian Institute of Health and Welfare and Australian Bureau of Statistics. The health and welfare of Australia's Aboriginal and Torres Strait Islander peoples. Canberra: Commonwealth of Australia, 1999.

4. Commonwealth Department of Health and Aged Care and Australian Institute of Health and Welfare. National Health Priority Areas Report: Cardiovascular Health. Canberra: Commonwealth Department of Health and Aged Care and Australian Institute of Health and Welfare, 1999.

5. Commonwealth Department of Health and Aged Care and Australian Institute of Health and Welfare. National Health Priority Areas Report: Diabetes Mellitus. Canberra: Commonwealth Department of Health and Aged Care and Australian Institute of Health and Welfare, 1998.

6. Public Health Division. The health of the people of NSWReport of the Chief Health Officer 2000. Sydney: Department of Health, 2000.
7. Colagiuri S, Colagiuri R, Warrd J. National Diabetes Strategy and Implementation Plan. Canberra: Diabetes Australia, 1998.

8. De Courten M, Hodge A, Dowse G et al. Review of the epidemiology, aetiology, pathogenesis, and preventability of diabetes in Aboriginal and Torres Strait Islander populations. Canberra. Commonwealth Department of Health and Family Services, 1998.

9. Marmot MG, Bosma H, Hemingway H, Brunner E, Stansfield S. Contribution of job control and other risk factors to social variations in coronary heart disease. Lancet 1997; 350: 235239.

10. Wilkinson R. Socioeconomic determinants of health: health inequalities relative or absolute standards. BMJ 1997; 314: 591.

11. Tennant C, McLean L. Mood disturbances and coronary heart disease: progress in the past decade. MJA 2000; 172: 151-152.

12. Roper NA, Bilous W, Kelly WF et al. Excess mortality in a population with diabetes and the impact of material deprivation: longitudinal, population based study. $B M J$ 2001;332: 1389-1393.

13. Cass A, Cunningham J, Wang Z, Hoy W. End Stage Renal Disease in Indigenous Australians: A disease of disadvantage. Darwin: Menzies School of Health Research. Presentation to NSW Department of Health, April 26, 2001 (unpublished).

14. Gilbert R, Jasik M, De Luise M, et al. Diabetes and Hypertension: Australian Diabetes Society position statement. MJA 1995; 163: 372-375.

15. O'Dea K. Westernisation, insulin resistance, and diabetes in Australian aborigines. MJA 1991; 155: 258-264.

16. Greenfield JR, Chisholm DJ. Clinical trials and clinical practice-bridging the gaps in type 2 diabetes. Aust NZJ Med. 2000; 30:483-491.

17. American Diabetes Association. Clinical Practice Recommendations, 1996. Diabetes Care 1996; 19(Suppl): S1-S118.

18. National Public Health Partnership Group. Preventing Chronic Disease: A Strategic Framework. Background Paper. Melbourne: National Public Health Partnership, 2001.

19. Northern Territory Preventable Chronic Disease Strategy, September, 1999: www.nt.gov.au/nths/cdc/preventable/ index.shtml.

20. UK Prospective Diabetes Study Group. Tight blood pressure control and risk of macrovascular and microvascular complications in Type 2 diabetes. BMJ 1998; 317: 703-713.

21. UK Prospective Diabetes Study Group. Intensive blood glucose control with sulphonylureas or insulin compared with conventional treatment and risk of complications in patients with Type 2 diabetes. Lancet 1998; 352: 837-853.

22. Hoy W et al. Reducing premature death and renal failure in Australian Aboriginals. A community based cardiovascular and renal protective program. MJA 2000; 172: 473-47.

23. McDermott R. Improving diabetes care in the primary health care setting: a randomised cluster trail in remote indigenous communities. MJA 2001; 174: 497-502.

24. Mitchell P, Pipemeyer J, Glass M, Mazze R, Gohdes D, Bradley R. Long-term impact of Staged Diabetes Management to improve metabolic control in an American Indian community. Diabetes 1998; 47 (Suppl 1): A183.

25. Policy Division. Aboriginal Vascular Health Resource Catalogue. Sydney, NSW Department of Health, 2002. 